\title{
Isolation of Gallic Acid and Estimation of Total Phenolic Content in Some Medicinal Plants and Their Antioxidant Activity
}

\author{
Giri R. Genwali ${ }^{1}$, Padam P. Acharya ${ }^{1}$ and Meena Rajbhandari ${ }^{2}$ \\ ${ }^{1}$ Central Department of Chemistry, Tribhuvan University, Kathmandu \\ ${ }^{2}$ Research Center for Applied Science and Technology \\ Tribhuvan University, Kathmandu \\ e-mail: karmacharyameena@gmail.com
}

\begin{abstract}
Gallic acid was isolated from ethylacetate soluble portion of the methanol extract of fruit pulp of Terminalia chebula and characterized by comparing the melting point, $\mathrm{R}_{\mathrm{f}}$ values, UV and IR spectra with authentic gallic acid. Quantitative determination of phenolics from different extracts of medicinal herbs such as Adhatoda vasica Nees, Bergenia ciliata (Haw) Sternb, Phyllanthus emblica Linnaeus, Terminalia bellirica (Gaerth) Roxb, Terminalia chebula Retzius and Vitex negundo Linnaeus which are commonly used as home herbal remedies for the primary health care was carried out using Folin-Ciocalteu colorimetric method. Gallic acid was used as the standard for the estimation of phenolics. All the investigated plant extracts contained high amount of phenolics but the highest amount was detected in 70\% acetone extract of B. ciliata (357.08 $\mathrm{mg} \mathrm{GAE} / \mathrm{g}$ sample) and the lowest amount was detected in 50\% aqueous methanol extract of $T$. bellirica (108.69 $\mathrm{mg} \mathrm{GAE/g} \mathrm{sample).} \mathrm{The} \mathrm{antioxidant} \mathrm{activity} \mathrm{of} \mathrm{selected} \mathrm{extracts}$ was determined by 1,1-diphenyl-2-picryl-hydrazyl (DPPH) assay. The extract having the highest phenolic content showed the lowest $\mathrm{IC}_{50}$ demonstrating the positive correlation between radical scavenging activity and total phenolic content.
\end{abstract}

Key words: antioxidant activity, gallic acid, medicinal herbs, total phenolic content

\section{Introduction}

Natural products, especially plants are the sources of different classes of phytochemicals. Phenolics are aromatic compounds bearing one or more hydroxyl substituents. Plant phenolics include phenolic acids, flavonoids, tannins, stillbenes and lignins. Phenolic acids are divided into derivatives of benzoic acid such as gallic acid and derivative of caeffic acid such as coumaric, caeffic and ferulic acids. Both flavonoids and phenolic acids are the most common polyphenols in our diet and distributed widely in fruits, vegetables, cereals and beverages.

In recent years, much attention has been paid by nutritionist on the dietary polyphenols due to their potent antioxidative effects and their credible effects in the prevention of various oxidative stress associated diseases. Oxidation process is one of the most important ways for producing free radicals in food and even in living systems. Free radicals cause many human diseases like cancer, Alzheimer's, cardiac, kidney and liver diseases, fibrosis, atherosclerosis, arthritis, neurodegenerative disorders and aging (Halliwell \& Gutteridge 1990). The preventive effects of plant polyphenols and their use in treating diseases are deduced from the epidemiological data as well as in vitro and in vivo studies (Art \& Hollamn 2005).

Many research studies have demonstrated that medicinal plants, fruits, and vegetables contain various phytochemicals with antioxidant activity, which are responsible for their beneficial health effects (Scalbert $e t$ al. 2005). In addition to vitamin C, E and carotenoids, polyphenols have shown strong antioxidant capacity (Rica-Evan et al. 1995). Due to their natural origin, the antioxidants obtained from plants are of greater benefit in comparison to synthetic ones such as butylatedhydroxy anisole (BHA) and butylatedhydroxy toluene(BHT). 
Nepal is rich in medicinal plants and we consume several types of these plants and plant products as herbal medicine for primary health care. These plants could be the potential source of natural antioxidants. However, no quantitative information are available on the polyphenol content and antioxidant effect of Nepalese herbs. In our previous study, we have quantified the total flavonoid content in different parts of Camellia plants and assessed their antioxidant activity (Acharya et al. 2013). The present study was conducted to quantify the total phenolic content in different medicinal plants using gallic acid as the standard. The antioxidant activity of selected extracts were determined using DPPH free radical scavenging assay. The correlation between total phenolic content and antioxidant activity was established. This will help to find new sources of safe and inexpensive natural antioxidants to use them in food or nutraceutical and pharmaceutical preparations to replace synthetic antioxidants.

\section{Methodology}

\section{Chromatographic materials and chemicals}

TLC foils (precoated) Silica gel $60 \mathrm{GF}_{254}, 0.2 \mathrm{~mm}$ and TLC foils (precoated) Cellulose F, $0.2 \mathrm{~mm}$, were purchased from Merck, Darmstadt, Germany. Sephadex LH-20 was purchased from Pharmacia Biotech, Uppasala, Sweden. Gallic acid was purchased from Merck, Germany and DPPH from Sigma Chemical Company, USA. Folin-Ciocalteu reagent was purchased from SD Fine Chemical Limited, Mumbai. All other chemicals were of analytical grade.

\section{Plant materials}

The dried fruits of $T$. chebula were purchased from the local market of Kathmandu. Other plants materials were collected from Gulmi district at an altitude between 1200-1600 m in March 2012. The plants were authenticated by Prof. R. P. Chaudary, Central Department of Botany, Tribhuvan University. Voucher specimens were deposited at the Research Centre for Applied Science and Technology, Tribhuvan University, Kathmandu, Nepal.

\section{Extraction of T. chebula fruits and isolation of gallic acid}

The flesh of dried fruits of $T$. chebula were ground to a fine powder $(100 \mathrm{~g})$ and extracted with $250 \mathrm{ml}$ methanol in a Soxhlet extractor for 15 hours. The extract was dried under reduced pressure at a Rotavapour to get viscous mass (36 g) which was suspended in 100 $\mathrm{ml}$ distilled water and then extracted with hexane (300 $\mathrm{ml})$ followed by ethyl acetate $(300 \mathrm{ml})$ in a separatory funnel. The solvent was evaporated in a rotavapour under reduced pressure to get hexane (5 g) and ethylacetate ( $9 \mathrm{~g}$ ) extracts.

The ethyl acetate extract ( 9 g) was chromatographed on a Sephadex LH-20 column eluting with methanol. Altogether five major fractions were collected after monitoring by TLC for the presence of gallic acid $\left(\mathrm{R}_{\mathrm{f}}\right.$ 0.44 ) in solvent systems, toluene-ethylacetate-formic acid (6:6:1). The fraction F-4 (200 mg) containing gallic acid was further purified by preparative thin layer chromatography in the solvent system tolueneethylacetate-formic acid (6:6:1). An amount of $20 \mathrm{mg}$ of sample F-4 was loaded on the home made preparative thin layer chromatographic plate (Silicagel $\mathrm{GF}_{254}, 20 \mathrm{x}$ $20 \mathrm{~cm}, 1 \mathrm{~mm}$ thickness). The band corresponding to gallic acid was scratched off. Silica gel was washed with methanol and the solvent was evaporated to get white crystals of gallic acid ( $3 \mathrm{mg}$ ). It was finally purified by re-crystallization with hot water.

\section{Preparation of extracts for the determination of total phenolics}

An amount of $20 \mathrm{~g}$ each of the dried and powdered plant materials was extracted with methanol $(200 \mathrm{ml})$ in a Soxhlet extraction apparatus for 10 hours. The residue was extracted with $50 \%$ aqueous methanol under reflux for 6 hours. Similarly, 20 g of each sample was percolated with $70 \%$ acetone and subjected to ultrasound-assisted extraction for 15 minutes. The extracts were filtered and the solvent was evaporated in a Rotary evaporator under reduced pressure.

\section{Determination of total phenolic content in different extracts \\ Preparation of standard}

The total phenolic content in plant extracts was determined by using Folin-Ciocalteu colourimetric method based on oxidation-reduction reaction (Waterhouse 2002). Various concentrations of gallic acid solutions in methanol (10, 25, 50 and $75 \mu \mathrm{g} / \mathrm{ml})$ were prepared. In a $20 \mathrm{ml}$ test tube, $1 \mathrm{ml}$ gallic acid of each concentration was added and to that $5 \mathrm{~mL}$ of Folin-Ciocalteu reagent (10\%) and $4 \mathrm{~mL}$ of $7 \% \mathrm{Na}_{2} \mathrm{CO}_{3}$ were added to get a total volume of $10 \mathrm{ml}$. The blue coloured mixture was shaken well and incubated for 
Giri R. Genwali et al/Isolation of Gallic Acid and Estimation.......

30 minutes at $40{ }^{\circ} \mathrm{C}$ in a water bath. Then the absorbance was measured at $760 \mathrm{~nm}$ against blank. All the experiments were carried out in triplicate. The average absorbance values obtained at different concentrations of gallic acid were used to plot the calibration curve.

\section{Preparation of sample}

Various concentrations of the extracts (25, 50, 100 and $200 \mu \mathrm{g} / \mathrm{mL}$ ) were prepared. Following the procedure described for standard, absorbance for each concentration of extract was recorded. Total phenolics content of the extracts was expressed as mg gallic acid equivalents (GAE) per gram of sample in dry weight $(\mathrm{mg} / \mathrm{g})$. The total phenolic contents in all samples was calculated the using the formula: $\mathrm{C}=\mathrm{c} \mathrm{V} / \mathrm{m}$ where, $\mathrm{C}=$ total phenolic content mg GAE/g dry extract, $\mathrm{c}=$ concentration of gallic acid obtained from calibration curve in $\mathrm{mg} / \mathrm{mL}, \mathrm{V}=$ volume of extract in $\mathrm{ml}, \mathrm{m}=$ mass of extract in gram.

\section{Statistical analysis}

All the experiments were carried out in triplicates and data reported are mean \pm standard deviation. Calculation of linear correlation coefficient and correlation analysis were carried out using MS Office Excel 2007. The linear regression equation for a straight line is, $\mathrm{Y}=\mathrm{mx}+\mathrm{c}$ where, $\mathrm{Y}=$ absorbance of extract, $\mathrm{m}$ $=$ slope of the calibration curve, $\mathrm{x}=$ concentration of extract, $\mathrm{c}=$ intercept. Using this regression equation, concentrations of extracts were calculated. From the calculated values of concentration of each extract, the total phenolics content was calculated.

Determination of antioxidant activity using 2, 2diphenyl-1-picrylhydrazyl free radical

Antioxidant activity of the selected extracts was assayed using DPPH free radical (Brand-Williams et al. 1995). DPPH solution (0.1 mM) was prepared by dissolving $3.9 \mathrm{mg}$ of DPPH in $100 \mathrm{~mL}$ methanol and stirred overnight at $4^{\circ} \mathrm{C}$. Thus prepared purple colored $\mathrm{DPPH}$ free radical solution was stored at $-20^{\circ} \mathrm{C}$ for further use.

Three different concentrations (5, 10 and $15 \mu \mathrm{g} / \mathrm{ml}$ ) of methanolic solutions of each extracts were prepared by the serial dilution of the stock solution $(10 \mathrm{mg} / \mathrm{ml})$ of the respective extract. To each $0.5 \mathrm{ml}$ extract solution, $2.5 \mathrm{ml}$ of $0.1 \mathrm{mM}$ DPPH solution was added.
A control was prepared by mixing $0.5 \mathrm{ml}$ distilled water and $2.5 \mathrm{ml} 0.1 \mathrm{mM}$ DPPH solution. These samples were shaken well and kept in dark for 30 minutes at room temperature. The absorbance was measured at $517 \mathrm{~nm}$ against the blank solution consisting $2.5 \mathrm{ml} \mathrm{MeOH}$ and $0.5 \mathrm{ml}$ distilled water. The radical scavenging activity was expressed as the radical scavenging percentage using the equation where; $A_{\mathrm{s}}=$ absorbance of sample solution, $A_{b}=$ absorbance of blank and $A_{C}=$ absorbance of control

$\%$ scavenging $=\left[\frac{\left(A s-A_{b}\right)}{A c}\right] \times 100$

$\mathrm{IC}_{50}$ value is the concentration of sample required to scavenge $50 \%$ of DPPH free radical and was calculated from the graph of radical scavenging activity against the concentration of extracts. Statistically, the correlation between antioxidant activity and total phenolic content was determined by plotting $\mathrm{IC}_{50}(\mu \mathrm{g} / \mathrm{ml})$ against TPC $(\mathrm{mg} / \mathrm{g})$.

\section{Results and Discussion Isolation and characterization of gallic acid from $T$. chebula}

Column chromatograph of the ethyl acetate phase of the methanol extract of T. chebula over Sephadex LH 20 followed by preparative thin layer chromatography on silicagel $\mathrm{GF}_{254}$ yielded gallic acid, which was purified by recrystallization with hot water. On TLC it appeared as dark coloured spot under UV $254 \mathrm{~nm}$, blue colour with ferric chloride reagent and folin reagent with $R_{f}$ value 0.4 in ethyl acetate-benzene (9:11) and 0.44 in toluene-ethyl acetate-formic acid (6:6:1). The melting point was found to be $248-250{ }^{\circ} \mathrm{C}$. Thin layer chromatography behavior and the melting point of the isolated gallic acid was in good agreement with the authentic gallic acid. The UV spectrum of the isolated and authentic gallic acid showed absorption bands at 220 and 270 $\mathrm{nm}$. The IR spectrum showed the band at 3495, 1666, $1612,1319,3278,1535,1419 \mathrm{~cm}^{-1}$. On the basis of all these facts, the compound isolated from T. chebula was confirmed as gallic acid.

Amount of different extracts obtained by different extraction methods

For the determination of total phenolics in plant extracts, different extraction approaches such as 
soxhlet using methanol, reflux using $50 \%$ aqueous methanol and ultra sound assisted using $70 \%$ acetone were applied. Generally methanol is used for the extraction of low molecular weight polyphenols, $50 \%$ aqueous methanol for the extraction of glycosides while $70 \%$ aqueous acetone is used for the extraction of higher molecular weight tannin components. Conventional long extraction time and high temperature increase the chance of oxidation of phenolics so ultrasound assisted extraction (UAE) technique was applied. The mechanism involves the propagation of the acoustic waves in $\mathrm{KHz}$ range that produce physical, chemical and mechanical effect causing disruption of biological membrane to facilitate the release of extractable compounds ( Loborde et al. 1998). The different extraction approaches provided different extracts in various amounts. The prepared extracts were used for the determination of total phenolics. The names of plants and the respective parts used for extraction and the amount of different extracts obtained per $20 \mathrm{~g}$ of dry plant materials under different extraction conditions are summarised in Table 1.

Table 1. Amount of different extracts obtained from $20 \mathrm{~g}$ of dried plant materials under different extraction conditions

\begin{tabular}{l|l|l|c|c|c}
\hline \multicolumn{1}{c|}{ Name of plants } & Parts used & Local name & $\begin{array}{c}\text { Methanol } \\
\text { extract (g) }\end{array}$ & $\begin{array}{c}50 \% \text { Methanol } \\
\text { extract (g) }\end{array}$ & $\begin{array}{c}70 \% \text { Acetone } \\
\text { extract (g) }\end{array}$ \\
\hline Terminalia bellirica (Gaerth) Roxb & Fruit & Baro & 2.0 & 5.0 & 3.5 \\
Terminalia chebula Retzius & Fruit & Harro & 3.5 & 5.0 & 3.0 \\
Phyllanthus emblica Linnaeus & Fruit & Amala & 1.5 & - & 1.5 \\
Bergenia ciliate (Haw) Sternb & Rhizome & Pashanveda & 5.5 & 2.5 & 3.0 \\
Adhatoda vasica Nees & Leaves & Kalovashak & 1.5 & 1.0 & 1.5 \\
Vitex negundo Linnaeus & Leaves & Simali & 2.0 & 2.0 & 3.0 \\
\hline
\end{tabular}

\section{Construction of calibration curve and calculation of total phenolic content in different plant extracts}

Total phenolic content in different extracts were determined by Folin-Ciocalteu (F-C) method using gallic acid as the standard. The absorbance values obtained at different concentrations of gallic acid was used for the construction of calibration curve (Fig 1). F-C method is based on the transfer of electrons in alkaline medium from phenolic compounds to phosphomolybedic phosphotungstic acid complexes to form blue coloured complexes, $\left(\mathrm{PMoW}_{11} \mathrm{O}_{40}\right)^{-4}$ that are determined spectrophotometrically at $760 \mathrm{~nm}$. Total phenolic content of the extracts was calculated from the regression equation of calibration curve $(\mathrm{Y}=0.014 \mathrm{x}$; $\left.\mathrm{R}^{2}=0.999\right)$ and expressed as $\mathrm{mg}$ gallic acid equivalents (GE) per gram of sample in dry weight (mg/g). The results are presented in Table 2.

Table 2. Total phenolic content in different extracts

\begin{tabular}{l|c|c|c}
\hline \multicolumn{1}{c|}{ Plant } & Methanol (mg/g) GAE & $\begin{array}{c}50 \% \text { Methanol }(\mathrm{mg} / \mathrm{g}) \\
\text { GAE }\end{array}$ & 70\% Acetone (mg/g) GAE \\
\hline T. chebula & $237.827 \pm 10.130$ & $318.361 \pm 10.370$ & $238.865 \pm 11.173$ \\
T. bellirica & $149.690 \pm 6.088$ & $108.692 \pm 9.914$ & $186.288 \pm 10.618$ \\
P. emblica & $197.371 \pm 4.244$ & - & $250.420 \pm 11.540$ \\
B. ciliata & $304.00 \pm 18.180$ & $117.428 \pm 9.316$ & $357.079 \pm 11.946$ \\
A. vasica & $109.512 \pm 9.589$ & $111.2 \pm 8.806$ & $186.495 \pm 10.570$ \\
V. negundo & $151.618 \pm 6.3886$ & $150.98 \pm 9.979$ & $226.518 \pm 11.0412$ \\
\hline
\end{tabular}




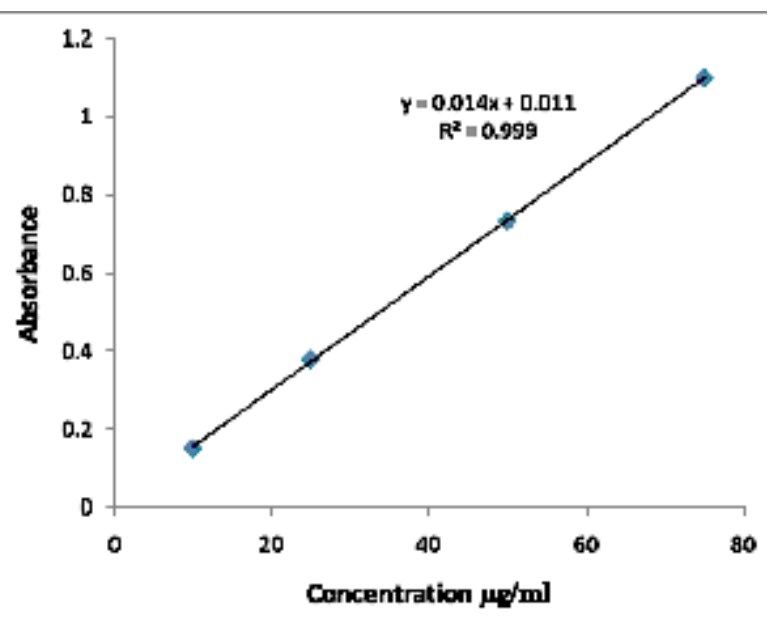

Fig 1. Calibration curve for standard gallic acid

In the case of $B$. ciliate, the highest amount of phenolic was detected in $70 \%$ acetone extract (357.08 mg GAE/ g sample) and in methanol extract (304.0 mg GAE/g sample) than in $50 \%$ aqueous methanol extract ( 117.43 mg GAE/g sample). In the case of $T$. chebula the highest amount of phenolics was detected in 50\% aqueous methanol extract (318.36 mg GAE/g sample) whereas the methanol and $70 \%$ acetone extracts contain the same amount of phenolics. Again, in the case of $P$. emblica (250.42 mg GAE/g), V. nigundo (226.51mg GAE/g sample), T. bellirica (186.28 mg GAE/g sample) and A. vasica (187.49 mg GAE/g sample) high amount of phenolics were detected in $70 \%$ acetone extracts than in methanol and 50\% aqueous methanol extracts. The lowest amount of phenolics were detected in 50\% aqueous methanol extract of $T$. bellirica (108.69 mg GAE/g sample). Comparing the extraction methods, $70 \%$ acetone is found to be the best solvent for the extraction of phenolics.

It is well known that the genus Bergenia is a high polyphenol, specially tannin containing plant (Rajbhandari \& Schoepke 2011). Previous phytochemical investigation of the rhizomes of $B$. ligulata led to the isolation of polyphenols like bergenin, 11-O-p-hydroxybenzoylbergenin, 6-Ogalloylarbutin and epiafzelechin (Rajbhandari et al. 2011).This could be the reason for the presence of high amount of phenolic in $70 \%$ acetone extract. On the other hand, T. chebula, one of the constituents of Ayurvedic formulation, triphala is a rich source of hydrolysable tannins, both gallotannins and ellagitannins and traditionally used to treat heart and spleen diseases. It was reported that $33 \%$ of total phyto-constituents of the fruits of $T$. chebula are hydrolysable tannins that are responsible for various pharmacological activity. These tannins contain phenolic carboxylic acid like gallic acid, ellagic acid, chebulic acid and gallotannins such as 1,6-di-O-galloylâ-D-glucose; 3,4,6-tri-O-galloyl-â-D-glucose; 2,3,4,6tetra-O-galloyl-â-D-glucose; 1,2,3,4,6-penta-O-galloyl$\hat{a}$-D-glucose. Ellagitannin such as punaclagin, casurarinin, corilagin and terchebulin and others such as chebulanin, neochebulinic acid, chebulagic acid and chebulinic acid are also reported (Chattopadhaya \& Bhattacharya 2007). Therefore, highest amount of phenolics were detected in 50\% methanol extract. Similarly, P. emblica is reported to contains phenolic compounds such as tannins, phyllaemblic compounds, gallic acid, ellagic acid, $1 O$-galloyl â D glucose, 3, 6 di $O$ galloyl D glucose, chebulinic acid, chebulagic acid, corilagin together with flavonoids, and vitamin C (Zhang et al. 2003). This could be the reason for the presence of high amount of phenolics in $70 \%$ acetone extracts. In $V$. negundo flavonoids and lignins were reported (Chandramu et al. 2003; Maurya et al. 2007) and it could be reason for high phenolic content in $70 \%$ acetone extracts. It is well known that $A$. vasica is a alkaloid containing plant but very few reports about polyphenol content are available (Kathale 2013) and in our finding high level of phenolic was detected in $70 \%$ acetone extract. T. bellirica is reported to contain $20 \%$ tannin, both condensed and hydrolysable; ethyl gallate, galloyl glucose, gallic acid, belliric acid and chebulic acid (Bele et al. 2010) were the main constituents. But the total phenolic content is found to be relatively low in all three extracts. Traditionally it is used to terat various ailments and also one of the constituents of triphala.

\section{DPPH radical scavenging assay}

The DPPH assay is based on the capability of an antioxidant to donate a hydrogen radical or an electron to DPPH radical, which is stable free radical with deep violet color. When an odd electron becomes paired in the presence of free radical scavenger of antioxidant agent, DPPH radicals get reduced to corresponding hydrazine, DPPH-H form (Piaxao et al. 2007) and the solution gets decolorized from its initial deep violet to light yellow colour. The degree of fall in the absorbance is measured spectrophotometrically and is proportional to the concentration of the antioxidant. 
Few extracts, $70 \%$ acetone extract $B$. ciliata having highest phenolic content, methanol extract of $T$. chebula having medium phenolic content and $50 \%$ methanol extract of $T$. bellirica having the lowest phenolic content were selected for their free radical scavenging capacities using DPPH free radicals. The absorbance values were measured at wavelength $517 \mathrm{~nm}$ for different concentration of extracts and the control. These values are used to calculate the percentage inhibitions of DPPHÿ radicals against the samples. The $\mathrm{IC}_{50}$ values of various extracts were calculated from the percentage inhibitions at various concentrations. The extract containing high amount of phenolic showed high radical scavenging activity. The results are shown in Table 3 .

Table 3. Total phenolic content and $\mathrm{IC}_{50}$ values

\begin{tabular}{l|l|l}
\hline Plart IExtacts & $\begin{array}{l}\text { Total } \\
\text { Pherolic } \\
(\mathrm{mg} / \mathrm{g}) \mathrm{GAE}\end{array}$ & $\begin{array}{l}\mathrm{IC}_{50} \\
\mu \mathrm{g} / \mathrm{ml}\end{array}$ \\
\hline T. chebuld Methanol extract & 237.827 & 109.78 \\
T. bellinic a/ $50 \%$ Methanol Extact & 108.692 & 213.11 \\
B. ciliate/ $70 \%$ Acetome Extract & 357.796 & 21.11 \\
\hline
\end{tabular}

The correlation between antioxidant activity and TPC (Fig. 2) had been determined by plotting $\mathrm{IC}_{50}(\mu \mathrm{g} / \mathrm{ml})$ against TPC (mg/g). A direct correlation between radical scavenging activity $\left(\mathrm{IC}_{50}\right.$ ) and TFC of the samples was observed which indicated that the phenolic compounds are responsible for DPPH free radical scavenging of the extracts.

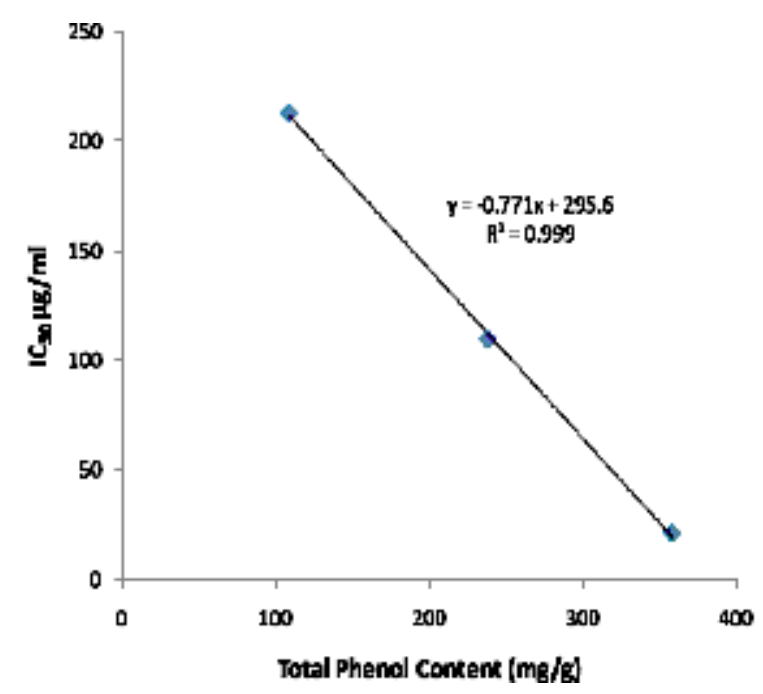

Fig. 2. correlation between DPPH radical scavenging activity and total phenolic
The results of the investigation indicated that the plants T. chebula, T. bellirica, $P$. emblica, B. ciliate, $A$. vasica and $V$. negundo that are often used as home herbal remedies are the rich source of high value polyphenol compounds. They are also the most commonly used components of Ayurvedic formulations. The consumption of these plants may have beneficial implications in human health such as in the treatment and prevention of cancer, cardiovascular disease and other pathologies by delaying or inhibiting the oxidation of lipids or other macromolecules and inhibiting the initiation or propagation of oxidative chain reactions. In addition, the easy availability of these plants make them promising sources of natural antioxidants and other bioactive compounds in food and pharmaceutical industries.

\section{Acknowledgements}

The authors would like to acknowledge Nepal Academy of Science and Technology, NAST for providing research grant for this work. They are grateful to Prof. U. Lindequist, University of Greifswald, Germany for providing authentic gallic acid and Sephadex LH 20 and Prof. S. M. Tuladhar, RECAST, T.U. for providing DPPH.

\section{References}

Acharya, P. P., G. R. Genwali and M. Rajbhandari. 2013. Isolation of catechin from Acacia catechu, estimation of total flavonoid content in Camellia sinensis and Camellia sinensis var. assamica collected from different geographical region and their antioxidant activity. Scientific World 11 (in press).

Art, I. C. and P. C. Hollman. 2005. Polyphenols and disease risk in epidemiological studies. American Journal of Clinical Nutrition 81: 317-325.

Bele, A. A., V. M. Jadar and V. J. Kadam. 2010. Potentials of tannins: A review. Asian Journal of Plant Science 9(4): 209-214.

Brand-William, W. Cuvelier and C. Berset. 1995. Use of a free radical method to evaluate antioxidant activity. Lebensmittel-wissenschaft und Technologie. 28 (1): 25-30.

Chandramu, C., R. D. Manohar, D. G. L. Krupadanam and R. V. Dashavantha. 2003. Isolation, characterization and biological activity of betulinic acid and ursolic acid from Vitex negundo L. Phytotherapy Research 17(2): 129-134.

Chattopadhaya, R. R. and S. K. Bhattacharya. 2007. Plant review Terminalia chibula. Pharmacognosy Review 23: $145-150$. 
Giri R. Genwali et al/Isolation of Gallic Acid and Estimation.......

Halliwell, P. C. and J. M. C. Gutteridge. 1990. Role of free radicals and catalytic metal ions in human disease: An overview. Methods in Enzymology 186: 1-85.

Kathale, S. V. 2013. Phytochemical screening and antimicrobial activity of ethanolic extracts of leaves of Adhatoda vasica Nees. International Journal of Pharmaceutical and Biological Sciences 4(1): 930 - 933.

Loborde, J. L., C. Bouyer, J. P. Caltagirone and A. Gkard. 1998. Acoustic bubble cavitation at low frequencies. Ultrasonics 36: 589-594.

Maurya, R., P. K. Shukla and K. Ashok. 2007. New antifungal flavonoid glycoside from Vitex negundo. Bioorganic \& Medicinal Chemistry Letters 17(1): 239-242.

Paixao, N., R. Perestrelo, J. C. Marques and J. S. Camara. 2007. Relationship between antioxidant capacity and total phenolic content of red, rose and white wines. Food Chemistry 105: 204-214.

Rajbhandari, M and Th. Schoepke. 2011. Characterization of the influenza virus inhibiting fractions of Bergenia ligulata. Nepal Journal of Science and Technology 12: 290-295.

Rajbhandari, M., H. R. Khatri, M. Lalk and M. B. Gewali and U. Lindequist, U. 2011. Phytochemical investigation and antibacterial activity of Bergenia ligulata. Journal Nepal Chemical Society 28: 110-114.

Rica-Evan, C. A., N. J. Miller, C.A. Bolwell and N. J. Bramley, P. M. Pridham. 1995. The relative antioxidant activities of plant derived phenolic flavonoids. Free Radical Research 22: 375-383.

Scalbert, A., C. Manach, C. Morand, C. Remesy and L. Jimenez. 2005. Dietary polyphenols and the prevention of diseases. Critical Review of Food Science and Nutrition 45:287-306.

Waterhouse, A. 2002. Determination of total phenolics. In: Current protocols in food analytical chemistry ( Ed Wrolstad, R. E). John Wiley and Sons, New York, Units I1.1.1-I1.1.8.

Zhang, L. Z., W. H. Zhao, Y. J. Guo, G. Z. Tu, S. Lin and L. G. Xin. 2003. Zhongguo Zhong Yao Za Zhi 28 (10): 940-943. 
Nepal Journal of Science and Technology Vol. 14, No. 1 (2013) 95-102 